\title{
Being a parent with a spinal cord injury
}

\author{
JJ Wyndaele \\ Antwerp, Belgium \\ E-mail: wyndaelejj@skynet.be
}

The main purpose of creating Spinal Cord 54 years ago, and its new online-only sister title Spinal Cord Series and Cases in 2015, was the desire to publish high-quality scientific research. Making research data and clinical experiences broadly available throughout the world is a secure and direct way to positively guide management and outcome of SCI, and these journals help to implement the goals of ISCoS as written in its Constitution and bylaws. The journals have become increasingly selective due to a high number of submissions, which leads to a high rejection rate. Manuscript rejection is often due to authors submitting content not directly related to SCI management. Sometimes manuscripts are re-directed from Spinal Cord to Spinal Cord Series and Cases when the content involves case reports, small series studies, and topics of local interest such as translations or observations of well-known facts in local hospitals. Spinal Cord Series and Cases is currently working toward building up enough content to qualify the journal for inclusion in PubMed and other search engines. We hope to achieve this very soon and will be submitting our application as soon as possible. Meanwhile, all Spinal Cord Series and Cases publications are fully available online with a Spinal Cord subscription, reaching the same libraries, institutions, and readership. We accept a large volume of high-quality papers, which inevitably leads to a longer period before they appear in print. However, the accepted manuscripts quickly become available online after acceptance in all search engines. Year after year we notice a continuous move from printed to online publications preferred by the readership of Spinal Cord. The future will thus probably lay in online publication. But guided by a survey of our members and readers, we will continue printing the journal as a substantial number prefer both online and print versions. This May 2016 issue has many interesting papers:

Review: Boades-Vaello et al. made a literature review on neuroplasticity after somatosensory injury with neuropathic pain. Sensitization and hyperexcitability of nociceptors and projection neurons of the dorsal horn, a strengthening in the descendent excitatory pathway and an inhibition of the descending inhibitory pathway of pain, are associated with molecular plastic changes such as overexpression of voltage-gated ion channels, algogen sensitive receptors and synthesis of several neurotransmitters. This knowledge guides the search for new pharmacological treatments.

Research methodology: van Middendorp et al. determined the top ten research priorities in SCI with a working group including professionals and consumers.

Animal studies: Mukhamedshine et al. studied two methods of glial cell-derived neurotrophic factor gene delivery into the spinal cord after traumatic injury. Aydemir et al. found in a study in the rat suggestion that melatonin could be an effective neuroprotective agent for treatment of SCI.

Clinical metabolic: Fatima et al. showed circadian variations in the circulating levels of serum cortisol and melatonin in patients with cervical SCI. Low levels of melatonin secretion during the night may contribute to a pervasive sleep disruption and increased pain perception. Citak et al. determined that CRP, serum alkaline and bone-alkaline phosphatase levels are no reliable screening methods for early heterotopic ossification. SadowskaKrepa et al. found that a voluntary participation in a long-term wheelchair rugby training program has several health promoting outcomes for individuals with chronic SCI.

Physical therapy: Sadeghi et al. report no significant difference in spasticity outcomes between static and dynamic standing training. Menedez Alegre et al. found a better outcome of popliteal artery blood velocity and skin temperature of the calf by simultaneously application of whole-body vibration and electromyostimulation.

Imaging: Suetomi et al. describe how diffusion tensor imaging values for the apparent diffusion coefficient might serve as a supplementary diagnostic indicator of the segmental levels of dysfunction in cervical spondylotic myelopathy.

Social participation/condition: Rasul and Biering-Sorensen provide insights into the challenges and satisfaction of being a parent post-SCI across various life domains. Arora et al. found the telephone based version of the SCI Secondary Conditions Scale reliable and valuable. Hata et al. examined the association between social participation and social support with self-rated health and dietary satisfaction.

Spinal Cord (2016) 54, 329; doi:10.1038/sc.2016.54 